\title{
Magnetic Field Induced Structural Transitions in 6CHBT-Based Ferronematics
}

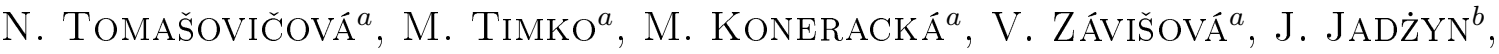 \\ E. BEAUGNON ${ }^{c}$, X. CHAUD $^{c}$ AND P. KOPČANSKÝ ${ }^{a}$ \\ ${ }^{a}$ Institute of Experimental Physics, Slovak Academy of Science, Watsonova 47, 04001 Košice, Slovakia \\ ${ }^{b}$ Institute of Molecular Physics, Polish Academy of Sciences, M. Smoluchowskiego 17, 60-179 Poznań, Poland \\ ${ }^{c}$ High Magnetic Field Laboratory, CNRS, 25 Avenue des Martyrs, Grenoble, France
}

\begin{abstract}
Ferronematics are stable colloidal suspensions of magnetic particles in nematic liquid crystals. The presence of the magnetic particles enhances the magnetic susceptibility of ferronematics, in comparison with pure liquid crystals. In this work the thermotropic liquid crystal 4 -( trans $-4^{\prime}-n$-hexylcyclohexyl)-isothiocyanato-benzene (6CHBT) and 6CHBT dissolved in phenyl isothiocyanate were doped with spherical magnetic nanoparticles with aim to increase the sensitivity of the liquid crystal on an external magnetic field. The volume concentration of the magnetic particles was $5 \times 10^{-4}$. The phase transition temperature from isotropic to nematic phase in the external magnetic field up to $12 \mathrm{~T}$ was monitored by precise capacitance measurements in the capacitance cells filled with nematic sample as well as with the prepared ferronematic sample. There was no observable shift in the transition temperature in the case of pure 6CHBT, 6CHBT doped with spherical particles and 6CHBT dissolved in phenyl isothiocyanate while in the case of 6CHBT dissolved in phenyl isothiocyanate doped with spherical magnetic particles the shift in the temperature about $9^{\circ} \mathrm{C}$ of the phase transition from isotropic to droplet state at the external magnetic field $12 \mathrm{~T}$ was observed.
\end{abstract}

PACS: 75.50.Mm, 61.30.Gd, 61.30.Hn

\section{Introduction}

Liquid crystals are anisotropic fluids that exhibit a wide variety of phases and phase transitions. Their ordering is a function of temperature. In nematic phase the molecules have no positional order, but tend to point in the same direction (along the director $\boldsymbol{n}$ ). Liquid crystal nanocomposites are considered to be extremely promising materials in which the properties of a liquid crystal phase used in different applications are modified by presence of various nanoparticles. There are many reports showing that doping of a nematic liquid crystal with small amount of nanoparticles affect the important properties of nematic materials resulting in a decrease of structural transitions threshold [1-5]. In the last two decades interest in these materials has grown substantially; not only because of the interesting physical problems, but also for the promise to provide an optical device technology based on magnetic switching. It has long been known that the possibility exists in liquid crystals for an external field to substantially alter the nematic-isotropic transition temperature [6-8]. However, the effect could not be induced by magnetic field $H$ [9] until recently [10]. The principal reason is that the estimated critical fields are well over $100 \mathrm{~T}$ for traditional liquid crystal materials [9].

The first experimental observation of the predicted magnetic-field dependence of the nematic-isotropic phase transition temperature has been recently carried out [10] on a powerful electromagnet ( $H$ up to $30 \mathrm{~T}$ ). To demonstrate the effect, besides the powerful electromagnet, the proper choice of a "non-traditional" (bent-core) nematic liquid crystal material was also necessary. The "non-traditional" nematic material chosen in Ref. [10], has considerably different physical properties from "traditional" calamitic nematics; the first-order character of the nematic-isotropic transition at the "clearing point" is substantially weaker than for "traditional" nematics. These properties, combined with the high magnetic field, have contributed to the observation of the phase transition temperature shift that was $\approx 0.8^{\circ} \mathrm{C}$ at the magnetic field of $30 \mathrm{~T}$. In our previous work [4] we have studied the structural transition in the 6CHBT-based ferronematics dissolved in phenyl isocyanate.

The aim of presented work was to investigate the magnetic field induced phase transition from isotropic to nematic state in 6CHBT-based ferronematics and 6CHBT dissolved in phenyl isothiocyanate and doped with spherical magnetic nanoparticles.

\section{Experiment}

The studied ferronematic samples were based on the thermotropic nematic 6CHBT 4-(trans- $4^{\prime}-n$-hexylcyclohexyl)-isothiocyanatobenzene. The 6CHBT is a low-melting enantiotropic liquid crystal with high chemical stability [11]. The temperature of the nematic-to-isotropic transition (clearing point) of the studied nematic is $T_{\mathrm{N}-\mathrm{I}}=42.8^{\circ} \mathrm{C}$. The nematic sample was doped with $\mathrm{Fe}_{3} \mathrm{O}_{4}$ particles (diameter $d \approx 10 \mathrm{~nm}$, standard deviation $\sigma=0.28$ ) coated with oleic acid as a surfactant. The magnetic particles were prepared by co-precipitation method [12]. The doping was simply done by adding this suspension, under continuous stirring, to the liquid crystal in the isotropic phase. Due to the small volume concentrations of the magnetic particles $\left(5 \times 10^{-4}\right)$ and surfactant in the prepared ferronematic samples the inter particle dipole-dipole interactions are avoided.

In the work of Kedziora et al. [13] the coexistence of 
nematic and isotropic phases was observed in the 6CHBT liquid crystal dissolved in a nonpolar medium (benzene) in the vicinity of the temperature of the isotropic-to-nematic transition. Due to the existence of a short-range orientational order of the mesogenic molecules (6CHBT), the pseudonematic domains (droplets of 500-1000 nm in size depending on the temperature) were formed in the isotropic phase. The size of these droplets increased, as the temperature of the liquid decreased. The temperature range of the droplet state depends on the molar fraction of the molar fraction of 6CHBT [4]. In our work liquid crystal 6CHBT was dissolved in phenyl isothiocyanate (that is more stable than benzene) and doped with spherical shaped $\mathrm{Fe}_{3} \mathrm{O}_{4}$ particles coated with oleic acid.

The structural transitions in ferronematic samples were indicated by capacitance measurements in a capacitor made of ITO-coated glass electrodes (LINCAM Co.). The capacitor with the electrode area approximately $1 \mathrm{~cm} \times 1 \mathrm{~cm}$ was connected to a regulated thermostat system, the temperature was stabilized with the accuracy of $0.05^{\circ} \mathrm{C}$. The distance between the electrodes (sample thickness) was $D=5 \mu \mathrm{m}$. The capacitance was measured at the frequency $1 \mathrm{kHz}$ by the high precision capacitance bridge Andeen Hagerling. The homogeneity and stability of the samples were verified by optical microscopy and by repeating the capacitance measurements after 5 months on the same samples, with reproducible results.

\section{Results and discussion}

The influence of the magnetic particles on the magnetic field induced isotropic-droplet state phase transition in "traditional" calamitic liquid crystal 6CHBT dissolved in phenyl isothiocyanate was studied by capacitance measurements. The used magnetic particles were spherical. The size of magnetic particles was much greater than the dimensions of the liquid crystal molecules, i.e. the magnetic particles can be regarded as macroscopic objects floating in the liquid crystal. The surface of the magnetic particles is able to orient the adjacent liquid crystal molecules due to strong orientational coupling between the magnetic particles and the liquid crystal matrix. There are two theoretical assumptions on the initial orientation of the director and the magnetic moment of the magnetic particles: perpendicular or parallel [14-16]. This theory considers the finite value of the surface density of anchoring energy $W$ at the nematic-magnetic particle boundary. The finite value of $W$, as well as the parameter $\omega$ that is defined as a ratio of anchoring energy to elastic energy of liquid crystal $(\omega=W d / K$, where $d$ is size of the magnetic particles and $K$ is orientational-elastic Frank modulus), characterize the type of anchoring of nematic molecules on magnetic particles surfaces. The parameter $\omega \gg 1$ characterizes the rigid anchoring. The soft anchoring, characterized by parameter $\omega \leq 1$, unlike the rigid, permits both types of boundary conditions $(\boldsymbol{m} \| \boldsymbol{n}$ and $\boldsymbol{m} \perp \boldsymbol{n}$ ). From our previous measurements on 6CHBT-based ferronematics we have obtained parameter $\omega=10^{-2}$, which characterizes soft anchoring and the boundary condition between $\boldsymbol{m}$ and $\boldsymbol{n}$ was found to be parallel [17]. In the case of parallel mutual initial orientation of the director $\boldsymbol{n}$ and the magnetic moment of the magnetic particles $\boldsymbol{m}$, the decrease of the critical magnetic field was observed.
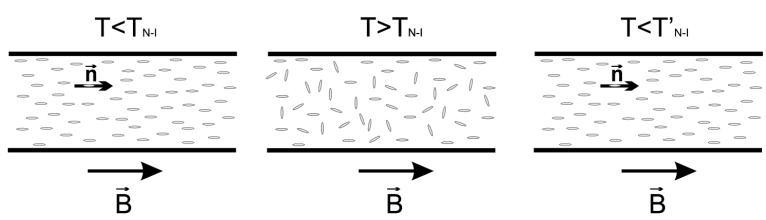

Fig. 1. Cross-section of the cell after application the constant magnetic field in the initial state, after heating and after cooling. The unit vector $\boldsymbol{n}$ (director) denotes the preferential direction of the nematic molecules.

During the measurements the magnetic field was applied parallel to the capacitor electrodes (see Fig. 1). The constant magnetic field was held, while the temperature was increased above the temperature of the transition from nematic to isotropic phase $T_{\mathrm{N}-\mathrm{I}}$ and then slowly decreased. The dependence of the measured capacitance reflects the re-orientation of the molecules of the liquid crystal to the nematic phase.

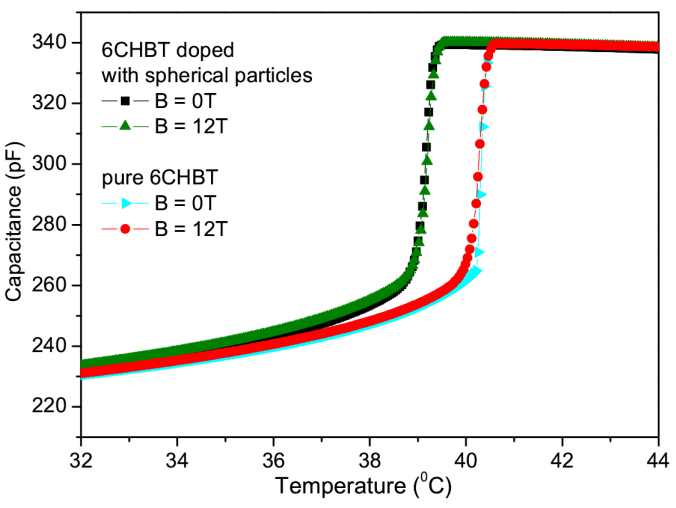

Fig. 2. Temperature dependence of the capacitance for pure 6 CHBT and 6 CHBT doped with magnetic particles at magnetic fields $B=0 \mathrm{~T}$ and $B=12 \mathrm{~T}$.

Figure 2 shows the temperature dependence of the capacitance for pure 6CHBT and 6CHBT doped with spherical particles measured without magnetic field and in magnetic field $12 \mathrm{~T}$. There is a shift in the temperature of isotropic to nematic phase transition due to doping but there is not observable shift in the magnetic field. The situation is different in the case of liquid crystal dissolved in phenyl isothiocyanate.

In our previous work [4], the structural phase transitions were studied in combined electric and magnetic fields and obtained results showed the shift in the temperature of phase transitions due to doping with magnetic particles. 


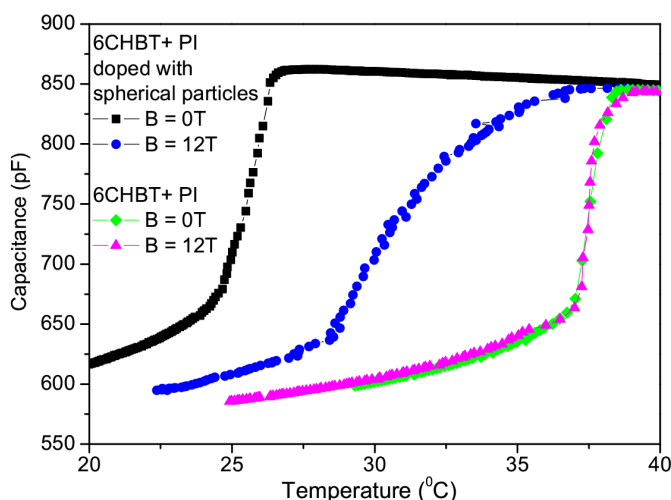

Fig. 3. Temperature dependence of the capacitance for 6CHBT dissolved in phenyl isothiocyanate (PI) (molar fraction 0.906) without magnetic particles and with magnetic particles at magnetic fields $B=0 \mathrm{~T}$ and $B=12 \mathrm{~T}$.

Figure 3 shows the temperature dependence of the capacitance for 6CHBT dissolved in phenyl isothiocyanate and 6CHBT dissolved in phenyl isothiocyanate doped with spherical particles measured without magnetic field and in magnetic field $12 \mathrm{~T}$. There is a shift in the temperature of isotropic to droplet state transition due to doping to the $28.5 \pm 0.05^{\circ} \mathrm{C}$. On the other hand, an additional shift in temperature of phase transition from isotropic to droplet state, as well as in the temperature of phase transition from droplet state to nematic phase in the external magnetic field of $12 \mathrm{~T}$. The magnetic field dependence of the temperature of the isotropic to droplet state phase transition is shown in Fig. 4.

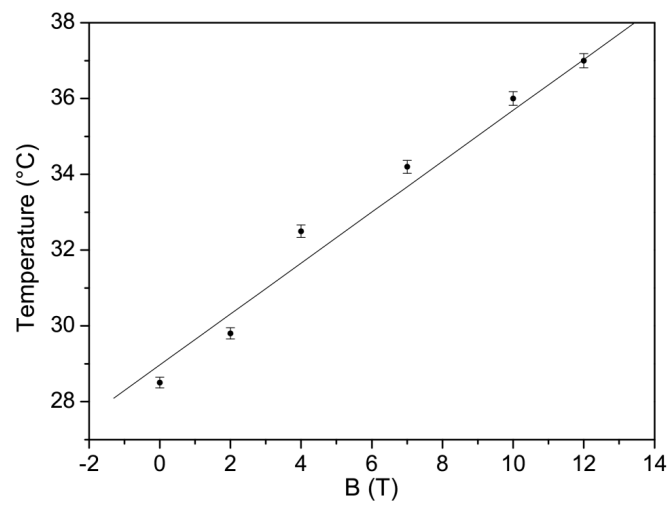

Fig. 4. Isotropic-droplet state phase transition temperature vs. applied magnetic field (line represents the best linear fit).

\section{Conclusion}

Our results showed that due to doping of liquid crystal 6CHBT dissolved in phenyl isothiocyanate with magnetic particles, the shift of the temperature of the phase transition from the isotropic to droplet state in the magnetic field up to $12 \mathrm{~T}$ is about $9{ }^{\circ} \mathrm{C}$.

\section{Acknowledgments}

This work was supported by the Slovak Academy of Sciences grant VEGA 0077 and Center of Excellence Nanofluid, APVV-0509-07 and APVV-0171-10, the Grenoble High Magnetic Field Laboratory, with support of EC from the 7th FP capacities "Transnational Access" - contract No. 228043 - EuroMagNET II, and with support by European Regional Development Fund project 26220120021.

\section{References}

[1] L.J. Martínez-Miranda, K. McCarthy, J.J. Harry, A. Noel, Appl. Phys. Lett. 89, 161917 (2006).

[2] O. Buluy, S. Nepijko, V. Reshetnyak, E. Ouskova, V. Zadorozhnii, A. Leonhardt, M. Ritschel, G. Schonhense, Y. Reznikov, Soft Matter 7, 644 (2011).

[3] P. Kopčanský, N. Tomašovičová, M. Koneracká, V. Závišová, M. Timko, A. Džarová, A. Šprincová, N. Éber, K. Fodor-Csorba, T. Tóth-Katona, A. Vajda, J. Jadzyn, Phys. Rev. E 78, 011702 (2008).

[4] N. Tomašovičová, P. Kopčanský, M. Koneracká, L. Tomčo, V. Závišová, M. Timko, N. Éber, K. Fodor-Csorba, T. Tóth-Katona, A. Vajda, J. Jadzyn, J. Phys. Condens Matter 20, 204123 (2008).

[5] P. Kopčanský, N. Tomašovičová, M. Koneracká, M. Timko, Z. Mitróová, V. Závišová, N. Éber, K. Fodor-Csorba, T. Tóth-Katona, A. Vajda, J. Jadzyn, E. Beaugnon, X. Chaud, Acta Phys. Pol. A 118, 988 (2010).

[6] W. Helfrich, Phys. Rev. Lett. 24, 201 (1970).

[7] I. Lelidis, G. Durand, Phys. Rev. E 48, 3822 (1993).

[8] H. Stanley, Introduction to Phase Transitions and Critical Phenomena, Oxford Science, New York 1971.

[9] C. Rosenblatt, Phys. Rev. A 24, 2236 (1981).

[10] T. Ostapenko, D.B. Wiant, S.N. Sprunt, A. Jákli, J.T. Gleeson, Phys. Rev. Lett. 101, 247801 (2008).

[11] R. Dabrowski, J. Dziaduszek, T. Szczucinski, Mol. Cryst. Liq. Cryst. Lett. 102, 155 (1984).

[12] P. Kopčanský, M. Koneracká, M. Timko, Potočová, L. Tomčo, N. Tomašovičová, V. Závišová, J. Jadzyn, J. Magn. Magn. Mater. 300, 75 (2006).

[13] P. Kedziora, J. Jadzyn, L. Hellemans, Phys. Rev. E 66, 021709 (2002).

[14] S.V. Burylov, Y.L. Raikher, J. Phys. Lett. A 149, 279 (1990).

[15] S.V. Burylov, Y.L. Raikher, J. Magn. Magn. Mater. 122, 62 (1993).

[16] S.V. Burylov, Y.L. Raikher, Mol. Cryst. Liq. Cryst. 255, 107 (1995).

[17] P. Kopčanský, N. Tomašovičová, M. Koneracká, V. Závišová, M. Timko, L. Tomčo, N. Éber, K. Fodor-Csorba, T. Tóth-Katona, A. Vajda, J. Jadzyn, E. Beaugnon, X. Chaud, Phys. Procedia 9, 82 (2010). 